\title{
Telomere length and possible link to X chromosome
}

Tim S Nawrot, Jan A Staessen, Jeffrey P Gardner, Abraham Aviv

\begin{abstract}
Summary
Background Because telomeres are eroded during mitosis, telomere length indicates the replicative history of human somatic cells. Clinical markers of ageing-such as pulse pressure and survival-are associated with telomere length. On the basis of findings of studies in twins, telomere length seems to be familial, but little is known about its mode of inheritance. We aimed to investigate the inheritance of telomere length.
\end{abstract}

Methods We measured terminal restriction fragment (TRF) length in white-blood-cell DNA taken from individuals from the family-based cohort of the Flemish Study on Environment, Genes, and Health Outcomes.

Findings We recorded no correlation in sex and age adjusted TRF length between spouses $(r=-0.05 ; p=0 \cdot 70)$ nor between fathers and sons $(r=-0 \cdot 16 ; p=0 \cdot 35)$. By contrast, we noted robust correlations in TRF length between fathers and daughters $(r=0.60 ; \mathrm{p}<0.0001)$; between mothers and sons $(r=0.41 ; \mathrm{p}=0.0017)$ and daughters $(r=0.59 ; \mathrm{p}<0.0001)$; and among siblings $(r=\geqslant 0 \cdot 61 ; p \leqslant 0 \cdot 0004)$.

Interpretation X-linked inheritance of TRF length is the most probable explanation for our findings. Pending confirmation, our observations suggest that the process of ageing might be an X-linked trait.

Lancet 2004; 363: 507-10
Study Coordinating Centre, Laboratory of Hypertension, Department of Molecular and Cardiovascular Research, University of Leuven, Campus Gasthuisberg, Herestraat 49, B-3000 Leuven, Belgium (T S Nawrot MSc, J A Staessen MD); and Hypertension Research Center, University of Medicine and Dentistry of New Jersey, New Jersey Medical School, Newark, NJ, USA

(J P Gardner PhD, Prof A Aviv MD)

Correspondence to: Dr Jan A Staessen

(e-mail: jan.staessen@med.kuleuven.ac.be)

\section{Introduction}

Telomeres consist of TTAGGG tandem repeats and telomere binding proteins that cap chromosomes and shield them from DNA-damage repair pathways. ${ }^{1}$ Telomeres undergo progressive attrition in cultured somatic cells because of the so-called end-replication problem. In utero, telomere length is similar in most tissues, ${ }^{2,3}$ but during extrauterine life, telomeres progressively shorten in proliferative somatic cells and their length diminishes with age. ${ }^{4-7}$ Telomere length is established by many factors, including cellular replicative history and telomerase - a reverse transcriptase consisting of an RNA component (hTR) and a catalytic protein component (hTERT) that has the ability to add telomere repeats to the ends of chromosomes. ${ }^{8}$ Based on studies in twins, telomere length seems to be familial, but little is known about its mode of inheritance. ${ }^{6,7}$ We aimed to investigate the inheritance of telomere length.

\section{Methods}

We enrolled into our study a random sample of families living in a geographically defined area of northern Belgium, who were part of the family-based cohort of the Flemish Study on Environment, Genes, and Health Outcomes. ${ }^{9}$ We undertook our study according to the principles outlined in the Helsinki declaration for investigation of human participants. The ethics committee of the University of Leuven approved the study, and participants gave written informed consent.

We took blood samples from all participants, from which we obtained DNA. We measured terminal restriction fragment (TRF) length in white-blood-cell DNA, as described. ${ }^{2}$ Briefly, we digested DNA samples with HinfI and RsaI and resolved them on 0.5\% agarose gels. We transferred DNA to a positively charged nylon membrane and detected telomeric DNA by Southern hybridisation to a digoxigenin $3^{\prime}$-end labelled 5'$(\mathrm{CCCTAA})_{3}$ after overnight incubation at $65^{\circ} \mathrm{C}$. We visualised labelled DNA with a digoxigenin luminescent detection procedure and by exposure on radiograph hyperfilm (Amersham Biosciences, Pistcataway, NJ, USA). We measured TRF length with ImageQuant (version 3.3; Amersham Biosciences) and SAS software (version 8.1; SAS Institute, Cary, NC, USA) as described. ${ }^{2}$ TRF length of every participant was the mean of duplicate measurements. If the duplicate for an individual varied by $5 \%$ or more we repeated the measurement and took the mean of the two that were less than 5\% apart. To control for possible variation between batches, we ran standards on every gel. Variation between batches was less than 3\%.

We used SAS software version 8.1 for database management and statistical analysis. We searched for possible covariables of TRF length by a stepwise regression procedure, with $\mathrm{p}$ values for independent variables to enter and stay in the model set at $0 \cdot 05$. To 
ascertain intrafamilial correlations, we ran generalised estimating equations as implemented in the PROC GENMOD procedure of the SAS package. We calculated correlation coefficients of TRF length between members of the same family as a measure of concordance (significant correlation) or discordance (non-significant correlation). Hence, in the context of this article, the term correlation and concordance are used interchangeably. We adjusted for confounders, treated pairs of relatives as clusters, and defined the working correlation matrix as unstructured. We applied Fisher's $Z$ transformation to compare correlation coefficients and to derive their significance.

\section{Role of the funding source}

The sponsors of the study had no role in study design, data collection, data analysis, data interpretation, writing of the report, or in the decision to submit for publication.

\section{Results}

Our multigeneration pedigrees spanned one $(n=13)$, two (10), or three (8) generations and included 128 parents and 199 offspring (83 sons and 116 daughters). Mean age of parents and offspring was 51.7 years (SD 12.2) and $30 \cdot 2$ years $(10 \cdot 9)$, respectively. From the pedigrees, we identified 34 father-son pairs, 47 father-daughter pairs, 51 mother-son pairs, and 71 mother-daughter pairs. On the basis of ABO blood group and rhesus phenotypes, we did not detect any cases of false paternity.

TRF length was normally distributed, with a mean length of $6.85 \mathrm{~kb}$ (SD 0.68). On stepwise regression analysis, sex, age, and smoking were significant determinants of TRF length, accounting for $1.2 \%$ $(p=0.036), 26.5 \%(p<0.0001)$, and $1.7 \%(p=0.011)$ of the variance, respectively. Rate of TRF length shortening was $0.024 \mathrm{~kb}$ per year in men (SE $0.003 ; \mathrm{p}<0.0001)$ and $0.019 \mathrm{~kb}$ per year in women $(0.003 ; \mathrm{p}<0.0001$; figure 1$)$. Age-adjusted TRF length was shorter in men $(n=119)$ than in women $(152 ; 6.77 \mathrm{~kb}$ [SE 0.05] vs 6.92 [0.05]; $\mathrm{p}=0 \cdot 028)$. Median daily tobacco consumption was 15 cigarettes (IQR 10-25) in 39 male smokers and 15 cigarettes $(10-20)$ in 43 female smokers. Sex and age

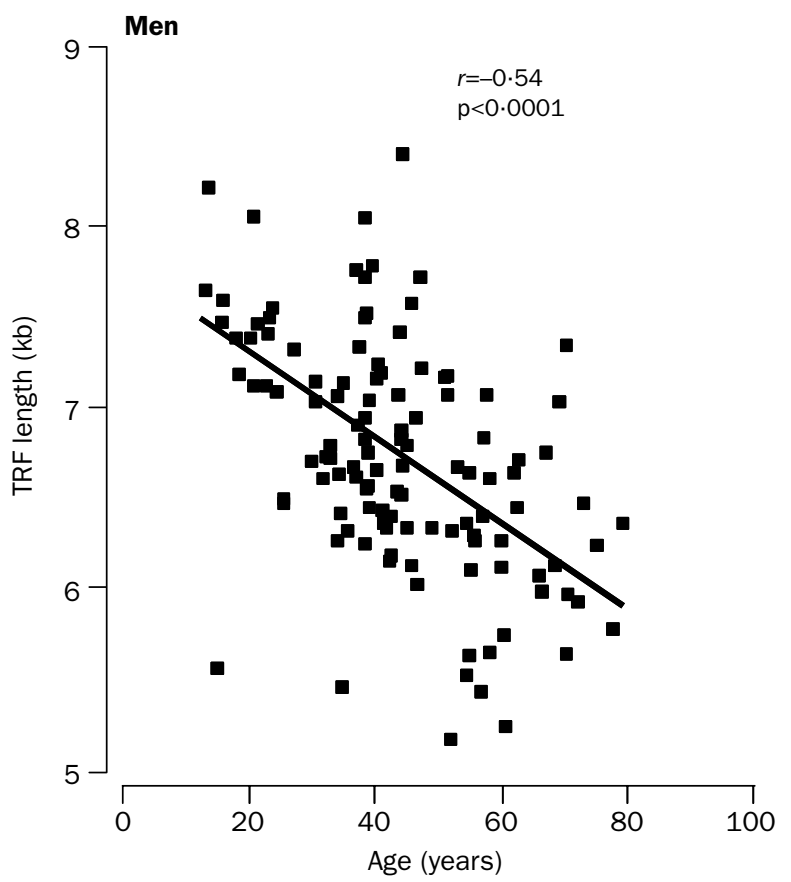

adjusted TRF length was lower in smokers $(n=82)$ than in non-smokers $(189 ; 6.72 \mathrm{~kb}[0.06]$ vs $6.91 \mathrm{~kb}[0.04]$; $\mathrm{p}=0 \cdot 014)$.

Both before (figure 2) and after (table) adjustment for sex, age, and smoking, spouse-spouse and father-son correlations were weak and not significant. By contrast, father-daughter, mother-son, and mother-daughter correlation coefficients were strong and significant. Intrafamilial concordance in TRF length was significantly higher in all mother-offspring relations than between spouses or between fathers and sons. Furthermore, strong concordance was noted in TRF length between offspring (table).

To assess the effect of outliers on our results, we did a sensitivity analysis, excluding individuals who were younger than age 40 years and had a TRF length of less than $6.0 \mathrm{~kb}$. With adjustments applied as before, the father-son correlation coefficient was $-0.02 \quad(p=0.90)$, the father-daughter correlation was $0.38 \quad(p=0.006)$, and the mother-son and mother-daughter correlations were $0.38(p=0.003)$ and $0.49(p<0.0001)$, respectively. Furthermore, to assess whether the significant differences between the father-son correlation coefficient and the other parent-offspring correlations might be due to a type I error, we randomly created fictive parentoffspring pairs, with the RANBIN function provided by the SAS software, with adjustments applied as before. The fictive mother-offspring correlation coefficient was $0.07(p=0.22)$, whereas the father-daughter and fatherson correlations were $0.02(\mathrm{p}=0.88)$ and $0.06(\mathrm{p}=0.70)$, respectively.

\section{Discussion}

The most plausible mechanism accounting for the high concordance of TRF length we noted between mothers and offspring and between fathers and daughters - but not between fathers and sons and between spouses-is an $\mathrm{X}$-linked mechanism of inheritance. Findings of sensitivity analyses, with outliers excluded or including fictive parent-offspring pairs, suggested that our results were not attributable to chance, but did not completely exclude this possibility.

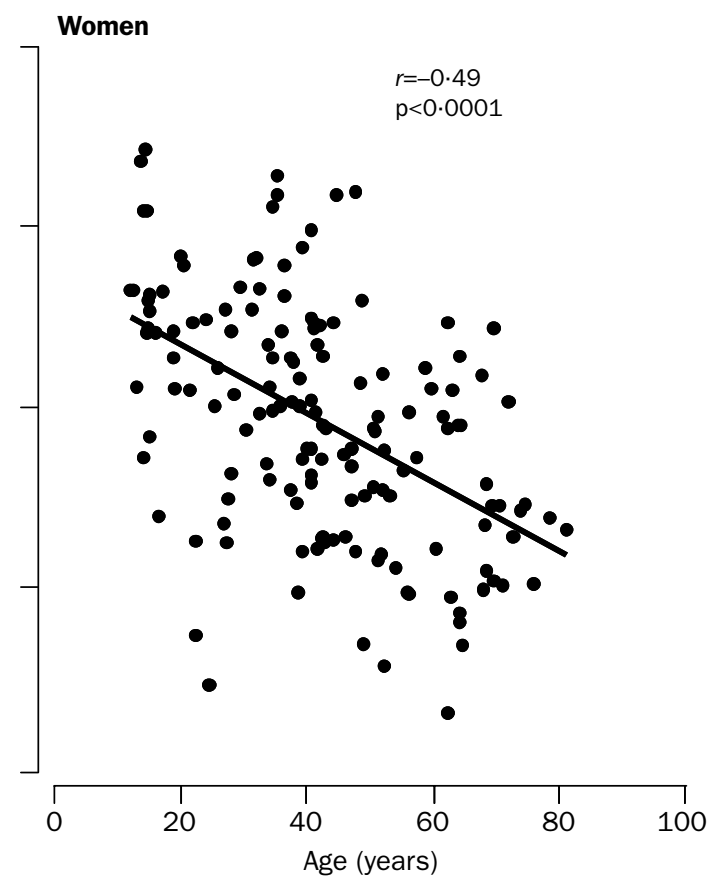

Figure 1: Relation between TRF length and age in men and women 

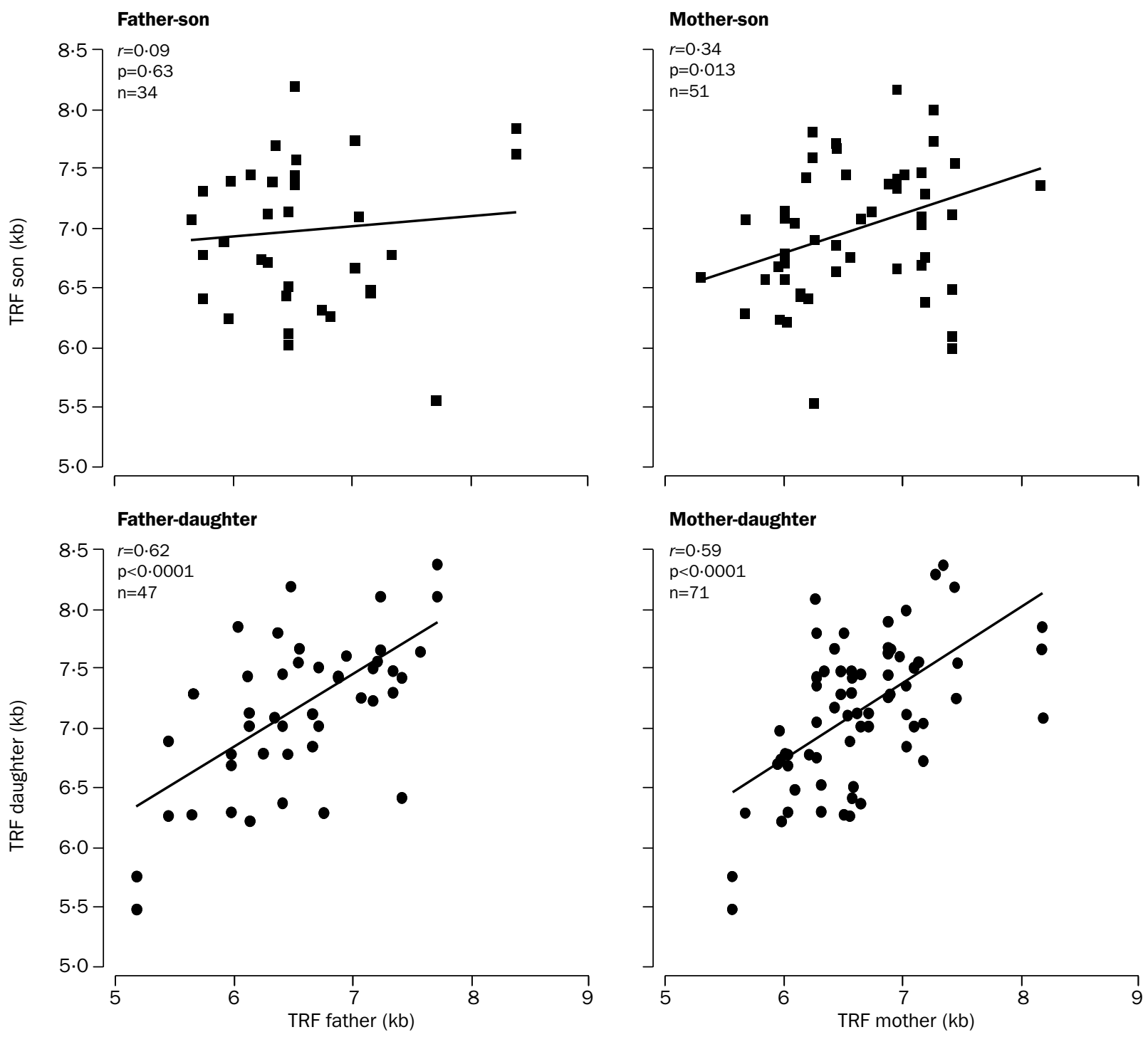

Figure 2: Intrafamilial relations for TRF length

The $\mathrm{X}$ chromosome harbours the $\mathrm{DKC} 1$ gene encoding the protein dyskerin, which is important for stable accumulation of the hTR component of telomerase. ${ }^{10}$ Cells from patients with $\mathrm{X}$-linked dyskeratosis congenita, caused by a missense mutation in the DKC1 gene, have decreased hTR concentrations, lower telomerase activity, and shortened TRF length. Thus, polymorphisms in the $D K C 1$ gene-with less profound effects than those noted in dyskeratosis congenita and possibly other yet unknown genes on the $\mathrm{X}$ chromosome - might be determinants of TRF length in the general population.

Oxidative stress has a role in cellular senescence and ageing. Nitric oxide activates telomerase and delays cell

\begin{tabular}{|c|c|c|c|}
\hline Relationship & Number of pairs & Partial $r$ & $\mathbf{p}$ \\
\hline Spouse-spouse & 64 & $-0.05 *$ & 0.70 \\
\hline Father-son & 34 & $-0 \cdot 16 *$ & 0.35 \\
\hline Father-daughter & 47 & $0.60 \dagger$ & $<0.0001$ \\
\hline Mother-son & 51 & $0.41 \dagger$ & 0.0017 \\
\hline Mother-daughter & 71 & $0.59 \dagger$ & $<0.0001$ \\
\hline Sister-sister & 22 & $0.61 \dagger \neq$ & 0.0004 \\
\hline Sister-brother & 25 & $0.71 \ddagger$ & $<0.0001$ \\
\hline Brother-brother & 23 & $0.83 \ddagger$ & $<0.0001$ \\
\hline
\end{tabular}

Correlation coefficients adjusted for sex (if applicable), age and smoking.

$* \uparrow \ddagger$ Partial correlation coefficients of similar size.

Intrafamilial correlation coefficients for TRF length

senescence. Thus, nitric oxide might react with cellular radicals and reduce oxidative stress, resulting in telomerase activation. It is noteworthy that the gene encoding the angiotensin II type 2 receptor (AGTR2)stimulation of which leads to enhanced nitric oxide production - also maps to the X chromosome. ${ }^{11}$

Mitochondrial DNA represents only a tiny fraction of the human genome. Mitochondria are implicated in modulation of oxidative stress and are almost completely of maternal origin. ${ }^{12}$ However, transmission of mitochondrial DNA would not account for the high father-daughter concordance in telomere length.

Our finding that age-adjusted TRF length is greater in women than in men accords with findings of previous studies. ${ }^{5,6}$ White-blood-cell TRF length is closely similar in newborn boys and girls, suggesting that sexual dimorphism in adult TRF length arises during extrauterine life. Several reasons might account for longer telomeres in women than in men. First, with advancing age, $\mathrm{X}$-inactivation in heterozygous women might be preferentially skewed to implicate the allele harbouring the genetic code that causes short telomeres. This occurrence was reported in carriers of $\mathrm{X}$-linked dyskeratosis congenita. ${ }^{13}$ TRF length of the inactive $\mathrm{X}$ chromosome is closely similar to that of the active 
$\mathrm{X}$ chromosome in normal newborn girls, but is shorter than the active $\mathrm{X}$ chromosome in older women. ${ }^{2}$ Second, an oestrogen-responsive element is present in hTERT, so the hormone can stimulate telomerase. ${ }^{14}$ Finally, reactive oxygen species accelerate telomere erosion at least in cultured cells. Women produce fewer reactive oxygen species and might metabolise them better than menperhaps because of oestrogen..$^{15}$ In this context, the shorter age-adjusted TRF length in smokers may also result from higher amounts of reactive oxygen species in the blood. ${ }^{16,17}$

Findings of several studies lend support to the idea that TRF length indicates biological ageing and that individuals with shorter telomeres than might be expected based on their chronological age are prone to disease. Indeed, TRF length was shorter in patients with atherosclerotic heart disease than in age-matched controls. ${ }^{18}$ Furthermore, independent of age and mean arterial pressure, arterial stiffness and pulse pressure were inversely correlated with TRF length in men. ${ }^{5}$ In a study stratified for sex and age, telomere length was a major independent predictor of all-cause mortality. ${ }^{19}$ Moreover, the mortality rate from infectious disease was eight times higher for individuals in the bottom $25 \%$ of the telomere length distribution than for those in the top $75 \% .{ }^{19}$ The hypothesis that genetic predisposition to chronic agerelated diseases might be associated with shortened telomeres and X-linked inheritance needs further investigation.

In conclusion, $\mathrm{X}$-linked inheritance of TRF is the most probable explanation for our findings. Pending confirmation, our observations suggest that the process of ageing might be an $\mathrm{X}$-linked trait.

Contributors

J A Staessen designed the study. T S Nawrot obtained data, constructed the database, and did the analysis. J A Staessen, T S Nawrot, and A Aviv wrote the first draft of the manuscript. J P Gardner supervised telomere measurements. All authors interpretated the results and prepared the final version of the manuscript.

Conflict of interest statement

None declared.

Acknowledgments

The Flemish Study on Environment, Genes, and Health Outcomes was supported by research grants from the Fonds voor Wetenschappelijk Onderzoek Vlaanderen, Brussels, Belgium (G.0424.03), the University of Leuven (OT/99/28), and the European Union (IC15-CT98-0329EPOGH and QLG-CT-2000-01137-EURNETGEN). Telomere research at the Hypertension Research Center of the University of Medicine and Dentistry of New Jersey, New Jersey Medical School was funded by NIH grant AG021593 and a grant from the Healthcare Foundation of New Jersey. We would like to thank Woodring E Wright (Dallas, USA) and Tim Spector (London, UK) for their constructive suggestions about this paper.

\section{References}

1 Blackburn EH. Switching and signaling at the telomere. Cell 2001; 106: $661-73$.

2 Okuda K, Bardeguez A, Gardner JP, et al. Telomere length in the newborn. Pediatr Res 2002; 52: 377-81.

3 Youngren K, Jeanclos E, Aviv H, et al. Synchrony in telomere length of the human fetus. Hum Genet 1998; 102: 640-43.

4 Allsopp RC, Vaziri H, Patterson C, et al. Telomere length predict replicative capacity of human fibroblasts. Proc Natl Acad Sci USA 1992; 89: 10114-18.

5 Benetos A, Okuda K, Lajemi M, et al. Telomere length as an indicator of biological aging: the gender effect and relation with pulse pressure and pulse wave velocity. Hypertension 2001; 37: 381-85.

6 Jeanclos E, Schork NJ, Kyvik KO, Kimura M, Skurnick JH, Aviv A Telomere length inversely correlates with pulse pressure and is highly familial. Hypertension 2000; 36: 195-200.

7 Slagboom PE, Droog S, Boomsma DI. Genetic determination of telomere size in humans: a twin study of 3 age-groups. Am f Hum Genet 1994; 55: 876-82.

8 Wong JMY, Collins K. Telomere maintenance and disease. Lancet 2003; 362: 983-88.

9 Staessen JA, Wang JG, Brand E, et al. Effects of three candidate genes on prevalence and incidence of hypertension in a Caucasian population. F Hypertens 2001; 19: 1349-58.

10 Mitchell JR, Wood E, Collins K. A telomerase component is defective in the human disease dyskeratosis congenita. Nature 1999; 402: 551-55.

11 Volpe M, Musumeci B, De Paolis P, Savoia C, Morganti A. Angiotensin II AT2 receptor subtype: an uprising frontier in cardiovascular disease? f Hypertens 2003; 21: 1429-43.

12 Vina J, Sastre J, Pallardo F, Borras C. Mitochondrial theory of aging importance to explain why females live longer than males. Antioxid Redox Signal 2003; 5: 549-56.

13 Vulliamy TJ, Knight SW, Mason PJ, Dokal I. Very short telomeres in the peripheral blood of patients with X-linked and autosomal dyskeratosis congenita. Blood Cells Mol Dis 2001; 27: 353-57.

14 Kyo S, Takakura M, Kanaya T, et al. Estrogen activates telomerase. Cancer Res 1999; 59: 5917-21.

15 Aviv A. Telomeres, sex, reactive oxygen species, and human cardiovascular aging. f Mol Med 2002; 80: 689-95.

16 Ayaori M, Hisada T, Suzukawa M, et al. Plasma levels and redox status of ascorbic acid and levels of lipid peroxidation products in active and passive smokers. Environ Health Perspect 2000; 108: 105-08

17 van Klaveren RJ, Demedts M, Nemery B. Cellular glutathione turnover in vitro, with emphasis on type II pneumocytes. Eur Respir 7 1997; 10: 1392-400.

18 Samani NJ, Boultby R, Butler R, Thompson JR, Goodall AH. Telomere shortening in atherosclerosis. Lancet 2001; 358: 472-73.

19 Cawthon RM, Smith KR, O'Brien E, Sivatchenko A, Kerber RA. Association between telomere length in blood and mortality in people aged 60 years or older. Lancet 2003; 361: 393-95. 